AIAA 2004-0463

Numerical Modeling of Pulse

Detonation Rocket Engine

Gasdynamics and Performance

C. I. Morris

NASAVGeorge C. Marshall Space Flight Center

TD40/Propulsion Research Center

Marshall Space Flight Center, AL USA 35812

42nd AIAA Aerospace Sciences Meeting and Exhibit

January 5-8, 2004/Reno, NV 


\title{
Numerical Modeling of Pulse Detonation Rocket Engine Gasdynamics and Performance
}

\author{
C. I. Morris* \\ NASAGeorge C. Marshall Space Flight Center \\ TD40/Propulsion Research Center \\ Marshall Space Flight Center, AL USA 35812
}

\begin{abstract}
Pulse detonation rocket engines (PDREs) offer potential performance improvements over conventional designs, but represent a challenging modeling task. 1 quasi-1-D, finite-rate chemistry computational fluid dynamics model for PDREs is described and implemented. Four different PDRE geometries are evaluated in this work: a baseline detonation tube, a detonation tube with a straight extension, and a detonation tube with two types of converging-diverging nozzles. The effect of extension length, and C-D nozzle area ratio, on the gasdynamics and performance of a PDRE is studied over a wide range of blowdown pressure ratios (1-1000). The overall effect of straight extensions and converging diverging nozales on performance and blowdown time is compared. The results are also compared to a steady-state rocket system using similar modeling assumptions.
\end{abstract}

\section{Introduction}

Pulse detonation engines (PDEs) have generated considerable research interest in recent years as a chemical propulsion system potentially offering improved performance and reduced complexity compared to conventional gas turbines and rocket engines. The detonative mode of combustion employed by these devices offers a theoretical thermodynamic advantage over the constant-pressure deflagrative combustion mode used in conventional engines. However, the unsteady blowdown process intrinsic to all pulse detonation devices has made realistic estimates of the actual propulsive performance of PDEs problematic. The recent review article by Kailasanath ${ }^{1}$ highlights some of the progress that has been made in comparing the available experimental measurements with analytical and numerical models.

The pulse detonation rocket engine (PDRE) ${ }^{2}$ has received a comparatively more limited research interest than the pulse detonation engines intended for airbreathing use. While conceptually similar, the rocket application of this technology requires investigation of the performance characteristics over a wide range of ambient pressures. In conventional rocket engine designs, the converging-diverging (C-D) nozzle plays a critical role in converting the thermal energy of the combustion products in the combustion chamber to directed kinetic energy in the exhaust. ${ }^{3}$ Thus, there has been considerable recent research interest in studying the use of nozzles for enhancing PDE performance. ${ }^{4}$ No attempt is made here to provide an exhaustive review of nozzle research for PDEs, but the most recent results, relevant to the rocket application and the current study, should be listed. Cooper and Shepherd ${ }^{5}$ have recently developed a partial-fill model for modeling the effect of straight extensions and diverging nozzles on detonation

\footnotetext{
* Research Engineer, Member AIAA.

This paper is a work of the U.S. Government and is not subject to copyright protection in the United Stares.
}

tubes at atmospheric conditions. This model makes use of the available experimental impulse data in the literature, and the partial-fill numerical simulations of $\mathrm{Li}$ and Kailasanath. ${ }^{6}$ Numerical studies of the effects of nozzles on a single detonation tube over a wider range of ambient pressures have been performed by $\mathrm{Cambier}^{7}$ and Mohanraj and Merkle. ${ }^{8}$ A study incorporating multiple tubes into a common nozzle was performed by Mohanraj et al. ${ }^{9}$ Talley and $\mathrm{Coy}^{10}$ have developed a O-D, constant- $\gamma$ model for studying the performance of an idealized constant-volume combustor with a nozzle over a wide range of pressure ratios.

In a previous paper by the author, ${ }^{11}$ parametric studies of the single-shot performance of a single, straight detonation tube were reported over a wide range of pressure ratios. A 1-D, unsteady method of characteristics code, employing a constant $\gamma$ assumption behind the detonation front, was developed for that study. Models of this type are computationally inexpensive, and may be used to illustrate the critical role of nozzles in the rocket PDE application. For example, a plot showing the specific impulse of various PDRE and steady-state rocket engine (SSRE) configurations as a function of blowdown pressure ratio is shown in Figure 1. The blowdown pressure ratio is defined throughout this work as the ratio of the initial fill (or combustion chamber) pressure, $\boldsymbol{P}_{c, i}$, to the ambient pressure, $P_{a}$. The calculations assume a propellant gas mixture of stoichiometric $\mathrm{H}_{2}-\mathrm{O}_{2}\left(P_{c, i}=1 \mathrm{~atm}, T_{c, i}=300 \mathrm{~K}\right)$. In each case, the final blowdown pressure at the end-wall in the PDRE simulations, $P_{c, f}$, is equal to $P_{a}$. Note that the SSRE performance calculations employ the same constant$\gamma$ assumption used in the PDRE calculations. There are four performance curves in the figure: a SSRE with a sonic nozzle, a SSRE equipped with an optimized C-D nozzle at each pressure ratio, a simple detonation tube (effectively a PDRE without a nozzle), and a PDRE fitted with a dynamically optimized C-D nozzle throughout the blowdown 


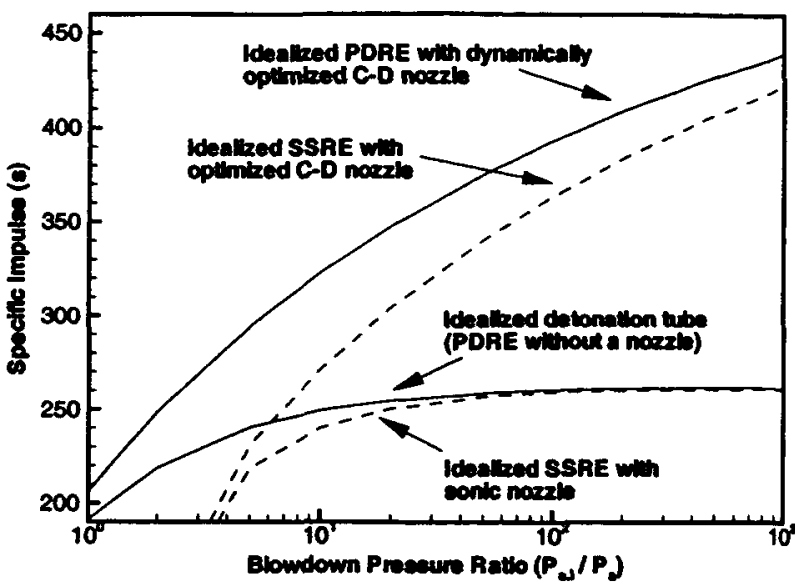

Fig. 1 Performance comparison of a pulse detonation rocket engine (PDRE) with a conventional steady-state rocket engine (SSRE) equipped with both sonic and optimized supersonic converging-diverging -nozales. The specific heat ratio, $\gamma$, is held constant in all models. The final blowdown pressure at the end-wall in the PDRE is equal to the ambient pressure $\left(P_{c, f}=P_{a}\right)$. Propellant mixture: stoichiometric $\mathrm{H}_{2}-\mathrm{O}_{2}$. Propellant initial conditions: $P_{c, i}=1 \mathrm{~atm}, T_{c, i}=300 \mathrm{~K}$.

process.

In examining the figure, a PDRE without a nozzle is perhaps best initially compared with a SSRE fitted with a sonic nozzle. Note that in this case the simple detonation tube provides superior specific impulse, compared to a SSRE with a sonic nozzle, over the entire range of pressure ratios. Note, however, that this advantage is relatively minor when expanding to the lowest ambient pressures. Moreover, in general a PDRE without a nozzle does not compare favorably to a SSRE fitted with an optimized C-D nozzle at blowdown pressure ratios above $\sim 7$. This result is primarily due to the largely-choked outflow from a simple detonation tube, as there is relatively little conversion of thermal energy to directed kinetic energy. However, it is also possible to calculate the performance of a detonation tube fitted with a dynamically optimized C-D nozzle throughout the blowdown process. This analysis effectively assumes quasi-steady flow within the nozzle at each time step in the simulation. The calculations show that if such a nozzle could be fitted to a PDRE, then the specific impulse of the device would exceed that of a comparable SSRE over the entire range of pressure ratios. While such a nozzle is a considerable idealization, these simple constant- $\gamma$ results clearly demonstrate the critical role that nozzles play in the performance of PDREs, particularly at the high pressure ratios typical for boost or in-space rocket applications.

The purpose of this paper is to study the effect of straight extensions and C-D nozzles on PDRE gasdynamics and performance, in greater detail than can be obtained using the previous constant- $\gamma$ method of characteristics approach. Details of the quasi-1-D, finite-rate chemistry computational fluid dynamics (CFD) model developed by the author are provided first. Four different PDRE geometries are evaluated: a baseline detonation tube, a detonation tube with a straight extension, and a detonation tube with two types of converging-diverging nozzles. The effect of extension length, and C-D nozzle area ratio, on the gasdynamics, performance, and blowdown time of a PDRE is studied over a wide range of blowdown pressure ratios (11000). The overall effectiveness of straight extensions and converging diverging nozzles in improving performance is evaluated. The results are also compared to a steady-state rocket system using similar modeling assumptions.

\section{Governing Equations}

The quasi-one-dimensional Euler equations are the governing model used in this study. A quasi-1-D model is at best an approximation of a pulse detonation or steadystate rocket engine. Real detonation waves exhibit clear multi-dimensional behavior. Additionally, flow separation in rocket engine nozzles is strongly dependent on multidimensional and viscous effects . However, as the primary goal of this study is to determine the effect of nozzles on pulse detonation rocket systems to first order, the quasi-1D Euler equations were deemed a reasonable model. The time-dependent form of these equations, including the effect of finite-rate chemistry, is written as follows:

$$
\frac{\partial \mathrm{U}}{\partial t}+\frac{1}{\mathrm{~S}} \frac{\partial \mathrm{FS}}{\partial x}=\mathrm{H}+\mathrm{W}
$$

where $S$ represents the cross sectional area of the duct, and is a function of distance, $x$. The state vector, $U$, and convective flux vector, $F$, are given by

$$
\mathrm{U}=\left[\begin{array}{c}
\rho_{1} \\
\rho_{2} \\
\vdots \\
\rho_{n s} \\
\rho u \\
\rho e
\end{array}\right], \quad \mathrm{F}=\left[\begin{array}{c}
\rho_{1} u \\
\rho_{2} u \\
\vdots \\
\rho_{n s} u \\
\rho u^{2}+p \\
u(\rho e+p)
\end{array}\right]
$$

The source vectors accounting for the effect of area change, $H$, and for the effect of finite-rate chemistry, $W$, are

$$
\mathbf{H}=\left[\begin{array}{c}
0 \\
0 \\
\vdots \\
0 \\
p \frac{\partial S}{\partial x} \\
0
\end{array}\right], \quad \mathbf{W}=\left[\begin{array}{c}
w_{1} \\
w_{2} \\
\vdots \\
w_{n s} \\
0 \\
0
\end{array}\right]
$$

Here, $\rho_{1}, \ldots, \rho_{n s}$ represent the mass densities of the $n s$ individual chemical species in the problem. The total gas density $\rho$ is sum of the individual species densities, $\rho=$ $\sum_{k=1}^{n s} \rho_{k} . u$ is the gas velocity in the $\mathrm{x}$ direction. $p$ is the gas pressure determined by the ideal gas law,

$$
p=\sum_{k=1}^{n s} \frac{\rho_{k}}{M_{k}} R T
$$

where $M_{k}$ is the molecular weight of the species, $R$ is the universal gas constant, and $T$ is the gas temperature. $e$ is 
the total energy per unit mass of the gas, $e=\epsilon+\frac{1}{2} u^{2}$, where $\epsilon$ is the specific energy per unit mass, and for the thermally perfect gas model considered here $\epsilon$ is a function of temperature and gas composition,

$$
\epsilon=\sum_{k=1}^{n s} Y_{k} \epsilon_{k}(T)
$$

where $Y_{k}$ represents the mass fraction of species $k, Y_{k}=$ $\rho_{k} / \rho$, and $\epsilon_{k}(T)$ is the specific energy per unit mass of species $k$, as a function of temperature. In this work, the ideal gas thermodynamic fits of $\mathrm{McBride}$ et al. ${ }^{12}$ are used to calculate the energy, specific heat, and Gibbs free energy for the 9 species in the problem $\left(\mathrm{N}_{2}, \mathrm{O}_{2}, \mathrm{H}_{2}, \mathrm{OH}, \mathrm{H}_{2} \mathrm{O}, \mathrm{H}\right.$, $\mathrm{O}, \mathrm{HO}_{2}$, and $\mathrm{H}_{2} \mathrm{O}_{2}$ ).

\section{Chemistry Model}

The source terms for finite-rate chemistry, $w_{k}$ in Eq. 3, are calculated from the sum of relevant chemical reactions for each species. Thus, for a chemical mechanism of $n r$ elementary reactions, each reaction is expressed as

$$
\sum_{l=1}^{n s} v_{l, n}^{\prime} X_{l} \rightleftharpoons \sum_{l=1}^{n s} v_{l, n}^{\prime \prime} X_{l}
$$

where the $v_{l, n}^{\prime}$ are the stoichiometric coefficients of species $l$ on the reactant side of reaction $n$, while the $\nu_{l, n}^{\prime \prime}$ are the corresponding stoichiometric coefficients on the product side. The production term for each species may be written as

$$
\begin{aligned}
w_{k} \equiv & \frac{\partial \rho_{k}}{\partial t}=M_{k} \sum_{n=1}^{n r}\left(v_{k, n}^{\prime \prime}-v_{k, n}^{\prime}\right) \\
& \cdot\left[k_{f} \prod_{l=1}^{n s}\left(\frac{\rho_{l}}{M_{l}}\right)^{v_{l, n}^{\prime}}-k_{b} \prod_{l=1}^{n s}\left(\frac{\rho_{l}}{M_{l}}\right)^{v_{l, n}^{\prime \prime}}\right]
\end{aligned}
$$

and $k_{f}$ and $k_{b}$ represent the forward and backward reaction rates, respectively. Details of the chemical kinetics mechanism for $\mathrm{H}_{2}-\mathrm{O}_{2}$ combustion used in this work are shown in Table 1. This mechanism was developed by Petersen and Hanson ${ }^{13}$ to model $\mathrm{H}_{2}-\mathrm{O}_{2}$ ignition at the elevated pressures typical of practical high-speed propulsion systems. The rate coefficients for reactions without pressure dependence take on the conventional Arrhenius form:

$$
k_{f}(T)=A T^{n} \exp \left(-E_{\text {act }} / R T\right)
$$

where $A$ is a constant, and $E_{\text {act }}$ is the activation energy in $\mathrm{cal} / \mathrm{mol}$. All reactions in the mechanism are reversible. The reverse reaction rate, $k_{b}$, is calculated from the forward rate (Eq. 8) and the equilibrium constant.

Pressure-dependent reactions are modeled using the approach given by Kee: ${ }^{14}$

$$
k_{f}=k_{\infty}\left[P_{r} /\left(1+P_{r}\right)\right] F
$$

where the reduced pressure, $P_{r}$, is

$$
P_{r}=\frac{k_{0}[\mathrm{M}]}{k_{\infty}}
$$

and the correction factor, $F$, is in the Troe ${ }^{15}$ form:

$$
\ln F=\left\{1+\frac{\ln P_{r}+c}{n-d\left(\ln P_{r}+c\right)}\right\}^{-1} \ln F_{c}
$$

The Troe centering parameter, $F_{c}$ is given by

$$
\begin{aligned}
F_{c}= & (1-a) \exp \left(-T / T^{* * *}\right)+a \exp \left(-T / T^{*}\right)+ \\
& \exp \left(-T^{* *} / T\right)
\end{aligned}
$$

where the constants $c=-0.4-0.67 \ln F_{c}, n=-0.75-$ $1.27 \ln F_{c}$, and $d=0.14$.

\section{Numerical Method}

The governing quasi-1-D Euler equations are solved in finite-volume form throughout the entire domain. The equations are solved in split form, in which a fluid convection subroutine solves Eq. 1 without the chemistry source vector $\mathbf{W}$,

$$
\frac{\partial U}{\partial t}+\frac{1}{S} \frac{\partial F S}{\partial x}=\mathbf{H}
$$

and a finite-rate chemistry integration subroutine solves Eq. 1 as a system of ordinary differential equations ignoring $F$ and $H$,

$$
\frac{\partial \mathrm{U}}{\partial t}=\mathrm{W}
$$

The code utilizes the Strang ${ }^{16}$ second-order time step splitting approach to couple the fluid convection and finiterate chemistry subroutines. For each complete time step, the chemistry subroutine is first called for one-half of a time step, followed by a full fiuid convection time step, and then followed by another half-step of the chemistry routine. Thus, in operator notation, the solution $U$ at time-step $n+1$ is

$$
\mathrm{U}^{n+1}=\mathcal{L}_{c}^{\Delta t / 2} \mathcal{L}_{f}^{\Delta t} \mathcal{L}_{c}^{\Delta t / 2} \mathrm{U}^{n}
$$

where $\mathcal{L}_{f}$ represents the fluid subroutine solving Eq. 13 and $\mathcal{L}_{c}$ represents the finite-rate chemistry subroutine solving Eq. 14. The time step splitting approach allows both high-quality fluid and chemical solvers to be developed and tested independently, and then joined together in relatively straightforward fashion. As described by Oran and Boris, ${ }^{17}$ time-step splitting works well when relatively small timesteps are used. Since the global time-step in the present code is strictly limited by the explicit Courant-FriedrichsLewy (CFL) condition, this is not thought to be a problem.

The fluid solver used here is the explicit, 2nd-order accurate (in time and space), symmetric-TVD algorithm described by Yee. ${ }^{18}$ The solver employs Roe's approximate Riemann solver modified for nonequilibrium ideal gases. ${ }^{19}$ It also incorporates suggestions by Larrouturou ${ }^{20}$ to ensure species positivity.

As with most reactive flow problems, the timeintegration of the chemistry mechanism in this work requires a stiff-ODE solver to ensure accuracy. The method used here employs a semi-implicit trapezoidal scheme as a predictor,

$$
\left[\mathbf{I}-\frac{1}{2} \Delta t \mathrm{~J}\right]\left(\mathrm{U}_{p}^{n+1}-\mathbf{U}^{n}\right)=\Delta t \mathbf{W}^{n}
$$


Table 1 Chemical kinetics mechanism for $\mathrm{H}_{2}-\mathrm{O}_{2}$ combustion, from Petersen and Hanson. ${ }^{13}$

\begin{tabular}{|c|c|c|c|c|c|}
\hline Number & Reaction ${ }^{a}$ & & $A^{b}$ & $n^{b}$ & $E_{x a t}^{b}$ \\
\hline 1 & $\mathrm{O}+\mathrm{H}_{2} \rightleftharpoons \mathrm{H}+\mathrm{OH}$ & & $5.00 \times 10^{4}$ & 2.70 & 6290 \\
\hline $2^{c}$ & $\mathrm{H}+\mathrm{O}_{2}+\mathrm{M} \rightleftharpoons \mathrm{HO}_{2}+\mathrm{M}$ & & $2.80 \times 10^{18}$ & -0.90 & $\mathbf{0}$ \\
\hline 3 & $\mathrm{H}+\mathrm{O}_{2}+\mathrm{O}_{2} \rightleftharpoons \mathrm{HO}_{2}+\mathrm{O}_{2}$ & & $3.00 \times 10^{20}$ & -1.70 & $\mathbf{0}$ \\
\hline 4 & $\mathrm{H}+\mathrm{O}_{2}+\mathrm{H}_{2} \mathrm{O} \rightleftharpoons \mathrm{HO}_{2}+\mathrm{H}_{2} \mathrm{O}$ & & $9.38 \times 10^{18}$ & -0.80 & $\mathbf{0}$ \\
\hline 5 & $\mathrm{H}+\mathrm{O}_{2}+\mathrm{N}_{2} \rightleftharpoons \mathrm{HO}_{2}+\mathrm{N}_{2}$ & & $2.60 \times 10^{19}$ & -1.20 & 0 \\
\hline 6 & $\mathrm{H}+\mathrm{O}_{2} \rightleftharpoons \mathrm{O}+\mathrm{OH}$ & & $8.30 \times 10^{13}$ & 0.00 & 14413 \\
\hline 7 & $\mathrm{H}+\mathrm{HO}_{2} \rightleftharpoons \mathrm{O}_{2}+\mathrm{H}_{2}$ & & $2.80 \times 10^{13}$ & 0.00 & 1068 \\
\hline 8 & $\mathrm{H}+\mathrm{HO}_{2} \rightleftharpoons \mathrm{OH}+\mathrm{OH}$ & & $1.34 \times 10^{14}$ & 0.00 & 635 \\
\hline 9 & $\mathrm{H}+\mathrm{H}_{2} \mathrm{O}_{2} \rightleftharpoons \mathrm{HO}_{2}+\mathrm{H}_{2}$ & & $1.21 \times 10^{7}$ & 2.00 & 5200 \\
\hline 10 & $\mathrm{OH}+\mathrm{H}_{2} \rightleftharpoons \mathrm{H}_{2} \mathrm{O}+\mathrm{H}$ & & $2.16 \times 10^{8}$ & 1.50 & 5200 \\
\hline \multirow[t]{2}{*}{$11^{d, e}$} & $\mathrm{OH}+\mathrm{OH}+\mathrm{M} \rightleftharpoons \mathrm{H}_{2} \mathrm{O}_{2}+\mathrm{M}$ & $k_{\infty}$ & $7.40 \times 10^{13}$ & -0.40 & $\mathbf{0}$ \\
\hline & & $k_{0}$ & $2.30 \times 10^{18}$ & -0.90 & -1700 \\
\hline 12 & $\mathrm{OH}+\mathrm{HO}_{2} \rightleftharpoons \mathrm{O}_{2}+\mathrm{H}_{2} \mathrm{O}$ & & $2.90 \times 10^{13}$ & 0.00 & -500 \\
\hline \multirow[t]{2}{*}{$13^{f}$} & $\mathrm{OH}+\mathrm{H}_{2} \mathrm{O}_{2} \rightleftharpoons \mathrm{HO}_{2}+\mathrm{H}_{2} \mathrm{O}$ & $k_{a}$ & $1.75 \times 10^{12}$ & 0.00 & 320 \\
\hline & & $k_{b}$ & $5.80 \times 10^{14}$ & 0.00 & 9560 \\
\hline \multirow[t]{2}{*}{148} & $\mathrm{HO}_{2}+\mathrm{HO}_{2} \rightleftharpoons \mathrm{O}_{2}+\mathrm{H}_{2} \mathrm{O}_{2}$ & $\boldsymbol{k}_{\boldsymbol{c}}$ & $1.30 \times 10^{11}$ & 0.00 & -1630 \\
\hline & & $k_{d}$ & $4.20 \times 10^{14}$ & 0.00 & 12000 \\
\hline $15^{h}$ & $\mathrm{O}+\mathrm{O}+\mathrm{M} \rightleftharpoons \mathrm{O}_{2}+\mathrm{M}$ & & $1.20 \times 10^{17}$ & -1.00 & $\mathbf{0}$ \\
\hline $16^{d}$ & $\mathbf{O}+\mathbf{H}+\mathrm{M} \rightleftharpoons \mathrm{OH}+\mathbf{M}$ & & $5.00 \times 10^{17}$ & -1.00 & 0 \\
\hline $17^{i}$ & $\mathrm{H}+\mathrm{OH}+\mathrm{M} \rightleftharpoons \mathrm{H}_{2} \mathrm{O}+\mathrm{M}$ & & $2.20 \times 10^{22}$ & -2.00 & $\mathbf{0}$ \\
\hline $18^{j}$ & $\mathbf{H}+\mathrm{H}+\mathrm{M} \rightleftharpoons \mathrm{H}_{2}+\mathrm{M}$ & & $1.00 \times 10^{18}$ & -1.00 & $\mathbf{0}$ \\
\hline
\end{tabular}

Note: species are $\mathrm{N}_{2}, \mathrm{O}_{2}, \mathrm{H}_{2}, \mathrm{H}_{2} \mathrm{O}, \mathrm{OH}, \mathrm{H}, \mathrm{O}, \mathrm{HO}_{2}, \mathrm{H}_{2} \mathrm{O}_{2}$.

"All reactions are reversible.

$b_{k_{f}}(T)=A T^{n} \exp \left(-E_{\text {act }} / R T\right)$; units are in cal, mol, $\mathrm{cm}^{3}$, and s.

$c_{M}$ does not include $\mathrm{O}_{2}, \mathrm{H}_{2} \mathrm{O}$, or $\mathrm{N}_{2}$; all collision efficiencies $=1.0$.

${ }^{d}$ Collision efficiencies for $\mathrm{M} ; \mathrm{H}_{2}=2.0, \mathrm{H}_{2} \mathrm{O}=6.0$, all others $=1.0$.

'Troe parameters: $a=0.7346, T^{* * *}=94, T^{*}=1756, T^{* *}=5182$.

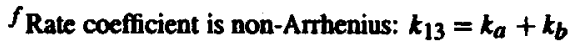

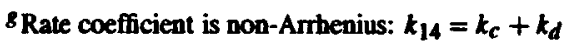

${ }^{h}$ Collision efficiencies for $\mathrm{M} ; \mathrm{H}_{2}=2.4, \mathrm{H}_{2} \mathrm{O}=15.4$, all others $=1.0$.

${ }^{i}$ Collision efficiencies for $\mathrm{M} ; \mathrm{H}_{2}=0.73, \mathrm{H}_{2} \mathrm{O}=3.65$, all others $=1.0$.

${ }^{j}$ Collision efficiencies for $\mathrm{M} ; \mathrm{H}_{2}=1.7, \mathrm{H}_{2} \mathrm{O}=7.0$, all others $=1.0$.

where $I$ is the identity matrix, $J$ is the Jacobian of $\mathbf{W}, J=$ $\partial W / \partial U$, and $U_{p}^{n+1}$ is the predicted next value of $U$. Newton iteration of the trapezoidal scheme is used as a corrector,

$$
\begin{aligned}
& {\left[\mathrm{I}-\frac{1}{2} \Delta t \mathrm{~J}\right]\left(\mathrm{U}_{c}^{n+1}-\mathrm{U}_{p}^{n+1}\right)=} \\
& \quad\left[\mathrm{U}^{n}-\mathrm{U}_{p}^{n+1}+\frac{1}{2} \Delta t\left(\mathrm{~W}^{n}+\mathrm{W}_{p}^{n+1}\right)\right]
\end{aligned}
$$

where $\mathbf{U}_{c}^{n+1}$ is the corrected next value of $\mathbf{U}$.

The chemistry integration subroutine is designed to use several sub-steps, if necessary for accuracy, within a given global half-step. The sub-steps are chosen such that no species concentration is predicted to change by more than $1 \%$ within each sub-step. Naturally, this leads to more extensive use of sub-steps when the concentration within a computational cell is far from equilibrium. The Jacobian J is evaluated analytically once per sub-step at $\mathbf{U}^{n}$. Thus, the LU decomposition of the bracketed matrix terms on the left hand side of $\mathrm{Eq} .16$ can be re-used in Eq. 17. Two corrector iterations were performed per sub-step.

\section{PDRE Simulations}

The four different PDRE geometries studied in this work (Fig.2) are highly idealized. Each is based on a constantarea $(2.0 \mathrm{~cm}$ diameter) detonation tube $20 \mathrm{~cm}$ in length. One end of the detonation tube is closed, while the other is either open to the environment (geometry A), attached to a straight extension (geometry B) or a converging-diverging nozzle section (geometries $C$ and D). Geometry A represents the baseline case. Four different straight extensions for geometry B were evaluated in this study: $L_{e}=4.0 \mathrm{~cm}$, $10 \mathrm{~cm}, 20 \mathrm{~cm}$, and $40 \mathrm{~cm}$. These extensions correspond to 
ratios of the total assembled tube volume, $V_{0}$, to the detonation tube volume, $V_{t}$, of $V_{0} / V_{t}=1.2,1.5,2.0$ and 3.0. In both of the converging-diverging nozzle geometries (C and $D$ ), the half-angle in the converging section is $15^{\circ}$. The difference between the two geometries is that in $\mathrm{C}$ the converging section is $0.4 \mathrm{~cm}$ in length, while in $D$ this length is $1.2 \mathrm{~cm}$. This results in a nozzle throat diameter for geometry $C$ of $1.786 \mathrm{~cm}$, and for $D$ of $1.357 \mathrm{~cm}$. Thus, the maximum rate of outflow is more restricted in geometry $D$ than in C, and in both cases is less than that of baseline geometry $\mathrm{A}$. The half-angle in the diverging section of the nozzle is fixed at $15^{\circ}$ in both geometries. The length of the diverging section, $L_{d}$, is a variable set at run-time, as detailed in the results and discussion to follow. The C-D nozzle area ratio is defined throughout this work as the ratio of the nozzle area at the exit plane to the throat area.

The detonation tube is pre-filled with a gaseous propellant mixture with no initial velocity. Stoichiometric $\mathrm{H}_{2}-\mathrm{O}_{2}$ at an initial pressure of $1 \mathrm{~atm}$, and initial temperature of $300 \mathrm{~K}$ is utilized for all calculations in this work. An idealized, massless diaphragm isolates the propellant mixture from the nozzle and ambient environment until ruptured by the detonation wave. The nozzle section is initially filled with $\mathrm{H}_{2}$ gas at the specified ambient pressure. The choice of the ambient gas has a nontrivial impact on the impulse of geometries B, C and D in single-shot simulations. Moreover, a number of options can be reasonably selected. Hydrogen is used here primarily due to its low molecular weight, which allowed a wide range of ambient conditions to be studied using the thermochemical model explained previously. The impact of using other possible choices will be addressed in a limited form in the results and discussion section.

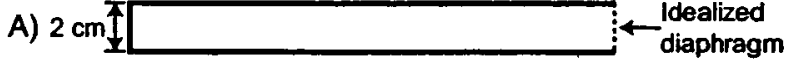

B)

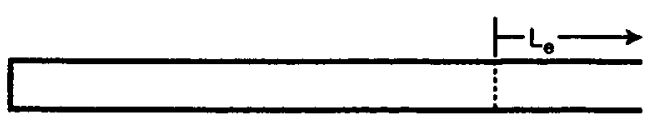

C)

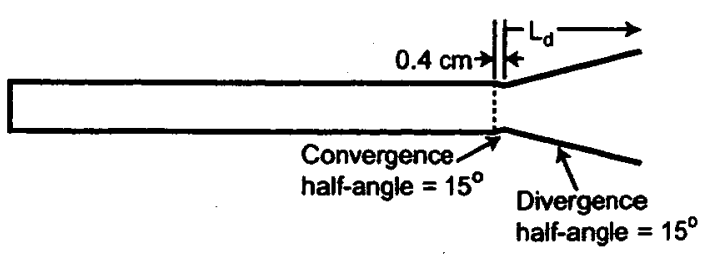

D)

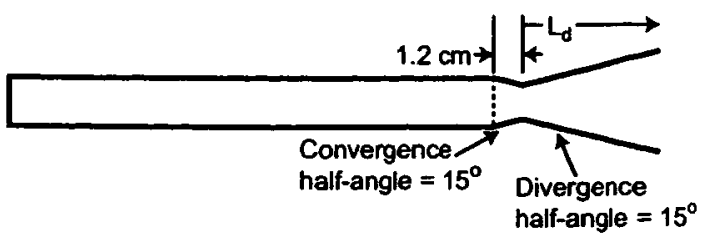

Fig 2 Schematic of PDRE geometries studied.
Detonation initiation is facilitated by calculating the equilibrium combustion conditions (computed at constant $\rho$ and $\epsilon$ ) for stoichiometric $\mathrm{H}_{2}-\mathrm{O}_{2}$ at $10 \mathrm{~atm}$ and $3000 \mathrm{~K}$, and then specifying these conditions in the cell adjacent to the closed end-wall.

Ghost cells are utilized to specify the boundary conditions in the problem. A reflection-type boundary condition is utilized at the closed end of the detonation tube to simulate a solid wall. The method of characteristics approach of Poinsott and Lele ${ }^{21}$ is used to calculate the exit flow boundary condition. For sonic (choked) or supersonic exit flow, all exit flow properties are determined by the interior flow. For subsonic exit flow, the ambient pressure is specified and the method of characteristics is used to compute the remaining flow properties. If reverse flow is detected at the exit, a subsonic inflow boundary condition is calculated using the ambient $\mathrm{H}_{2}$ gas as a constant pressure and enthalpy reservoir.

Two additional special restrictions are imposed in the simulations. In order to simulate the effect of an idealized diaphragm, only the detonation tube portion of the domain (from the closed wall to the diaphragm location) is computed initially. A reflection-type boundary condition is specified at the diaphragm location until the pressure in the adjacent cell rises $1.0 \%$ above the initial fill value. This special restriction is subsequently removed, and the entire domain is computed. Additionally, there is a check performed when the exit flow is supersonic. Since in this case the exit boundary conditions are entirely calculated from the interior flow, there is no way for the exit flow to return to a subsonic condition. ${ }^{8}$ Therefore, at each time step a check is made to determine if the pressure from standing normal shock at the exit is less than the ambient pressure. If true, then the normal shock properties are specified in the last interior cell, and a subsonic outflow boundary condition computed at the exit.

\section{Grid Resolution}

Grid resolution is a critical issue in all finite-rate chemistry CFD models. Due to the extremely fast kinetics of the $\mathrm{H}_{2}-\mathrm{O}_{2}$ system, numerical modeling of the reaction zone of undiluted $\mathrm{H}_{2}-\mathrm{O}_{2}$ Chapman-Jouguet (C-J) detonations requires an exceptionally fine grid spacing. The C-J detonation velocity of stoichiometric $\mathrm{H}_{2}-\mathrm{O}_{2}$ at an initial condition of $1 \mathrm{~atm}$ and $300 \mathrm{~K}$ is calculated by the NASA CEA thermochemical code ${ }^{22}$ to be $2837.1 \mathrm{~m} / \mathrm{s}$. Using the calculated frozen shock properties at this velocity, and the chemical kinetics mechanism described previously, the length of the C-J reaction zone for this mixture can be estimated as $\sim 0.02 \mathrm{~mm}$. Thus, a grid spacing of roughly $1 \mu \mathrm{m}$ would be required to resolve the reaction zone. This is not currently practical, nor is it necessary for the performance estimates of this study. The chief requirement here is that the grid resolution accurately calculate the C-J state and velocity.

A grid resolution comparison of pressure and temperature profiles of a propagating C-J detonation, $t=30 \mu \mathrm{s}$ 

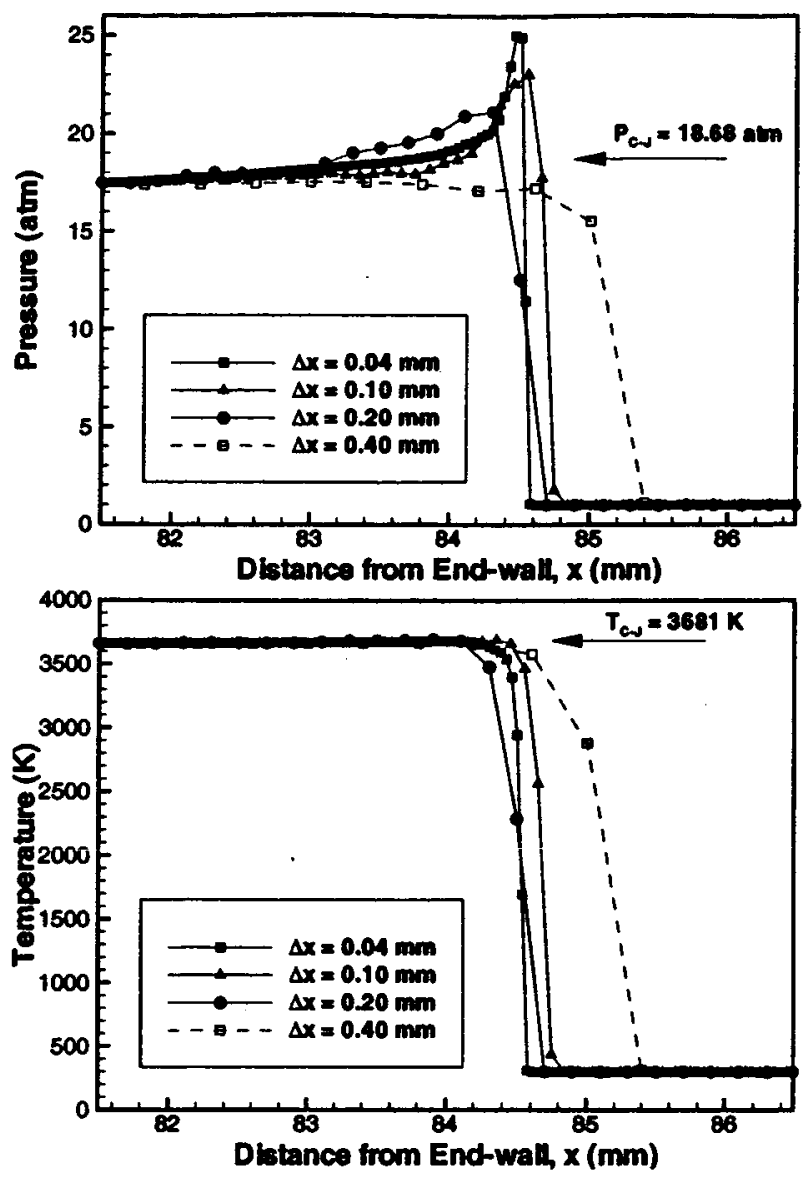

Fig. 3 Grid resolution comparison showing pressure (upper panel) and temperature (lower panel) profiles of a propagating $C$-J detonation wave at $t=30 \mu$ s after initiation.

after initiation, is shown in Fig. 3. The resolutions vary over one order of magnitude. As even the finest resolution here, $\Delta x=0.04 \mathrm{~mm}$, is twice the estimated reaction zone size, none of the resolutions can accurately capture the ignition process behind the leading shock wave. Rather, in all cases, the propagating $\mathrm{C}-\mathrm{J}$ detonation is smeared over several cells, resulting in the pressure spike and subsequent relaxation toward the C-J state in the finer grids. All four resolutions capture the detonation velocity with reasonable accuracy $(\sim 2836 \mathrm{~m} / \mathrm{s}$ for $\Delta x=0.04 \mathrm{~mm}, \sim 2840 \mathrm{~m} / \mathrm{s}$ for the other resolutions), though the precision of this estimate is naturally lower for the coarser grids. Note, however, that in two finer grids the pressure relaxes to the C-J value in a significantly shorter distance than in the coarser grids. Based on these observations, a uniform grid spacing of $\Delta x$ $=0.10 \mathrm{~mm}$ was utilized for all simulations in this study.

\section{Thrust Calculations}

The time-dependent thrust is calculated at each time step by two different methods. One measure of the thrust assumes a control volume tightly bounding the solid surfaces of the PDRE, and is determined by integrating the pressure difference across all surfaces. A second measure assumes a rectangular control volume encapsulating the PDRE. This measure of thrust is determined from the sum of the time rate of change of the internal momentum integral across the domain, the momentum flux from the nozzle section, and the pressure difference across the control volume. In general, there is excellent agreement between the two thrust calculations, and the time-integrated impulse calculations agree to within $0.1 \%$. In all simulations, the calculation proceeds until the pressure at the closed end-wall is equal to the ambient pressure $\left(P_{c, f}=P_{a}\right)$. Thus, these simulations should be thought of as single-shot results.

\section{Results and Discussion}

There are several critical factors which can impact the performance of a PDRE system. The blowdown pressure ratio is one of the most important parameters governing the performance of any rocket-type system. Additionally, the length of a straight extension, or the area ratio of a C-D nozzle, can have a large effect on PDRE performance. In the following section, the essential gasdynamics of the blowdown process in the four PDRE geometries are reviewed. This is followed by a parametric study of the effect of blowdown pressure ratio, extension length, and C-D nozzle area ratio on specific impulse and blowdown time. The relative effectiveness of straight extensions and C-D nozzles in improving performance is compared. A comparison of the performance of a PDRE fitted with an optimized C-D nozzle with an equivalent steady-state rocket engine is also provided.

\section{PDRE Blowdown Gasdynamics}

The blowdown process of a PDRE can be thought of as occurring in several different phases, typically differentiated by the location of important wave structures in the tube. Consider the simplest geometry, the baseline detonation tube (geometry A), at a blowdown pressure ratio of $10\left(P_{a}=0.1 \mathrm{~atm}\right)$. Pressure profiles, at several representative points in the.blowdown history, are shown for this case in the upper panel of Fig. 4. The detonation is initiated at the end-wall at $t=0$. The subsequent propagation of the detonation wave is clearly shown in the pressure profile labelled $t=60 \mu \mathrm{s}$. The sharp pressure rise associated with the detonation front is followed by a Taylor expansion wave which acts to slow and expand the combustion products behind the detonation front. Well behind the detonation front, the products reach a steady pressure at zero forward velocity. Once the detonation reaches the exit of the tube and breaks the idealized diaphragm, outflow begins, and an expansion wave propagates back upstream. This expansion wave expands and accelerates the combustion products toward the exit, and is visible in the $t=180 \mu \mathrm{s}$ profile. Once this expansion reaches the end-wall, it reflects and begins propagating back downstream toward the exit again ( $t=300 \mu$ s curve). Eventually, the expansion wave reaches the tube exit, and further reduces the pressure at that location $(t=420 \mu \mathrm{s})$. Note that the pressure at the end-wall is steady at a "plateau" value $\left(P_{3}\right)$ until the primary expansion wave reaches the end-wall. Similarly, the pressure at the exit plane also rapidly achieves a quasi- 

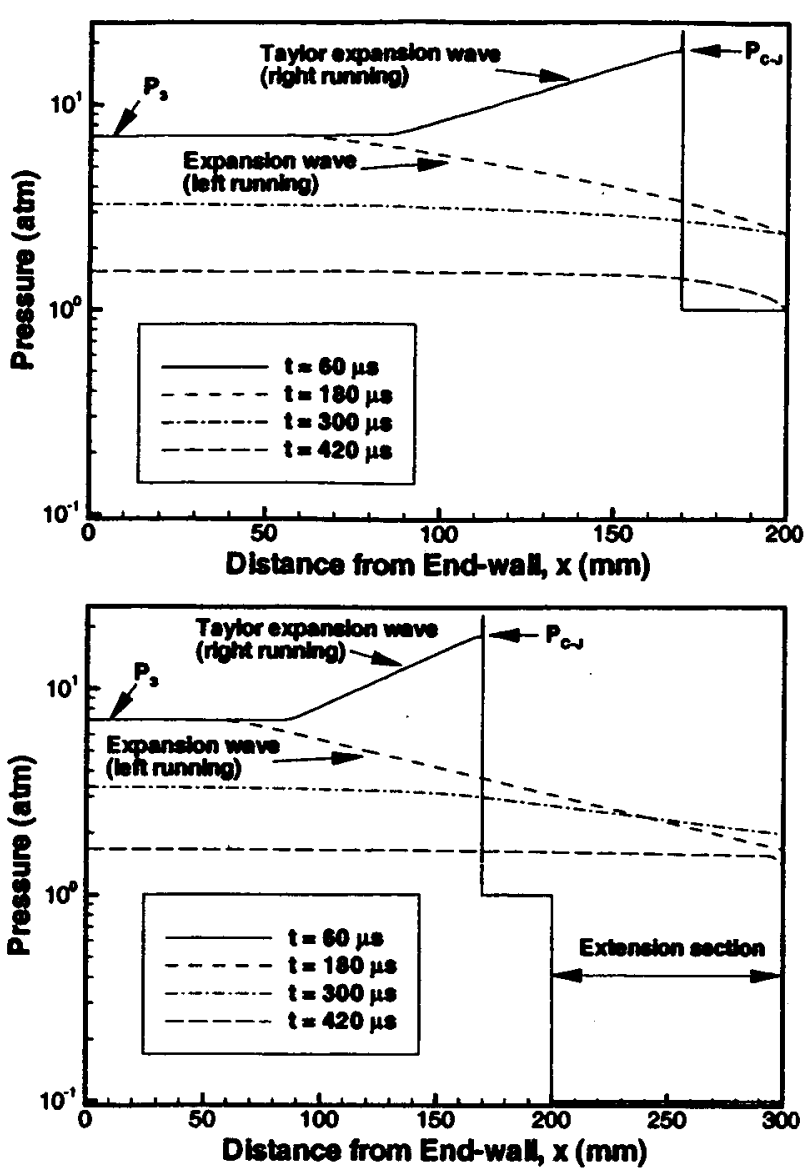

Fig. 4 Pressure profiles for PDRE geometry A (upper panel) and $B$ with $L_{\epsilon}=10 \mathrm{~cm}$ extension (lower panel) at representative points in the blowdown history. Blowdown pressure ratio is $10\left(P_{a}=0.1 \mathrm{~atm}\right)$. Propellant mixture: stoichiometric $\mathrm{H}_{2}$ $O_{2}$. Propellant initial conditions: $P_{c, i}=1 \mathrm{~atm}, T_{c, i}=300 \mathrm{~K}$.

steady value, which holds until the primary expansion wave again reaches the exit after reflection from the end-wall.

The addition of an extension to the detonation tube (geometry B) primarily serves to increase the amount of time required for some of the waves to traverse the tube. Pressure profiles at representative times in the blowdown history of a detonation tube with a $L_{e}=10 \mathrm{~cm}$ extension section are shown in the lower panel of Fig. 4. As above, the blowdown pressure ratio of this example case is 10 . As in the baseline case, a primary expansion wave is initiated when the detonation reaches the end of the detonation tube. Additionally, as there is no combustible gas mixture in the extension, the detonation wave changes into a transmitted shock wave in the extension. When the transmitted shock reaches the exit of the extension section, it initiates a secondary expansion wave which also travels upstream toward the end-wall. This leads to pressure profiles quite similar to the baseline case. However, the overall effect of the extension is to slow the rate of pressure relaxation at the end-wall.

A converging-diverging nozzle has a significant effect on the pressure distribution of a PDRE. Pressure profiles are shown for two C-D example cases in the upper (geome-
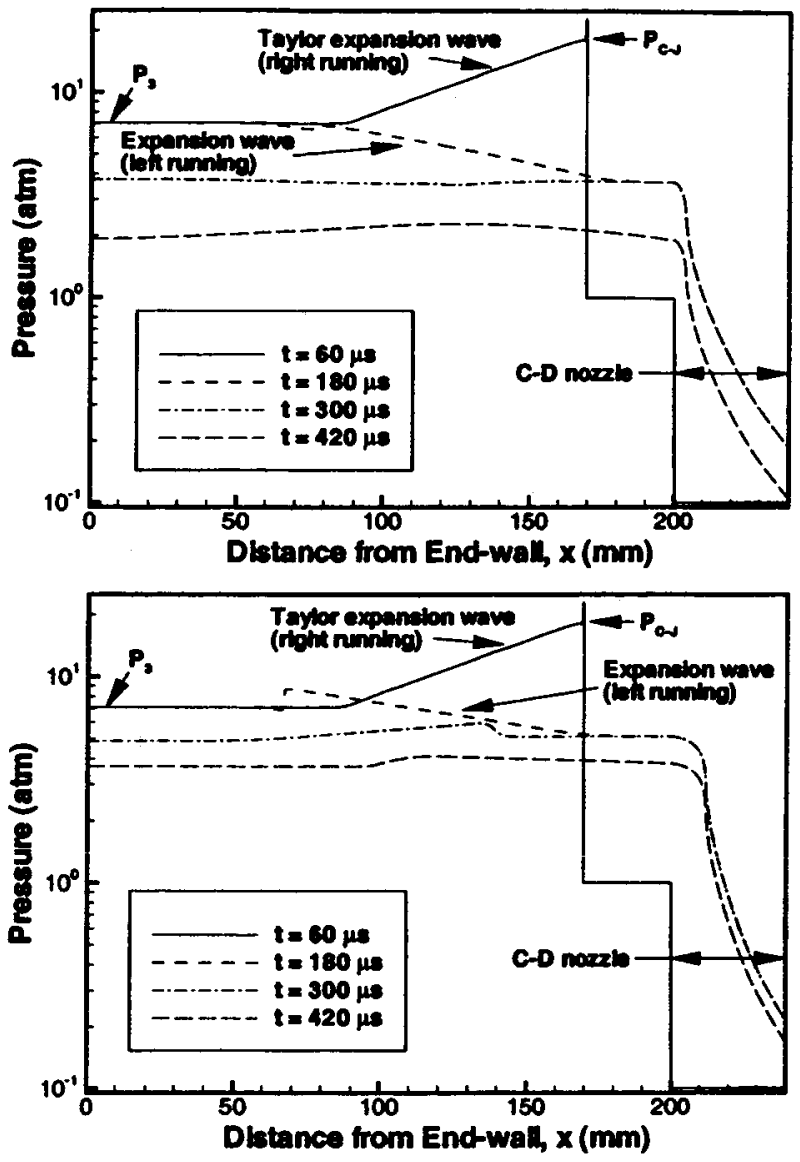

Fig. 5 Pressure profiles for PDRE geometry $C$ with $L_{d}=$ $3.6 \mathrm{~cm}$ (upper panel), and D with $L_{d}=2.8 \mathrm{~cm}$ (lower paned), at representative points in the blowdown history. Blowdown pressure ratio is $10\left(P_{a}=0.1 \mathrm{~atm}\right)$. Propellant mixture: statchiometric $\mathrm{H}_{2}-\mathrm{O}_{2}$. Propellant initial conditions: $P_{c, t}=1 \mathrm{~atm}$, $\mathrm{T}_{c, i}=300 \mathrm{~K}$.

try C with $L_{d}=3.6 \mathrm{~cm}$ ) and lower panels (geometry D with $L_{d}=2.8 \mathrm{~cm}$ ) of Fig. 5 . The blowdown pressure ratio of these cases is again 10. As was the case previously, a primary expansion wave, initiated when the detonation reaches the end of the detonation tube, is a key factor in controlling the pressure relaxation at the end-wall. However, it is clear from examining he figures that the C-D nozzle acts to expand the combustion products to a much lower exit pressure than would otherwise be accomplished by the detonation tube alone. The primary difference between the two C-D nozzle cases is the significantly smaller throat in geometry $\mathrm{D}$, which results in reduced mass outflow compared to geometry $\mathrm{C}$. This flow restriction is significant enough that the primary expansion wave is led by a weak shock wave, clearly visible in the lower panel of the figure.

The impulse history of all four of these example cases is shown in Fig. 6. The instantaneous thrust can be thought of as the slope of these impulse curves. As discussed previously, the primary effect of the extension in geometry $B$ is to reduce the rate of pressure relaxation at the end-wall. This results in a modestly higher thrust in the later portions 
of the blowdown history, compared to the baseline geometry $\mathrm{A}$. In contrast, the $\mathrm{C}-\mathrm{D}$ nozzle has a more significant effect on the thrust and impulse. Geometry $\mathrm{C}$ has a comparatively higher thrust and resultant impulse throughout the entire outflow portion of the blowdown history. Due to the more restricted mass outflow, and internal shock wave reflections, the instantaneous thrust in geometry D is generally lower than that of the other cases. However, although the overall blowdown time is significantly longer than geometry $\mathrm{C}$, the final impulse is nearly equivalent. One additional important observation which can be made from Fig. 6 is that typically the integrated impulse will achieve a value quite close to, or even slightly exceeding, the final value well before the end of the simulation. This is particularly true of the two C-D nozzle geometries, and is observed over the entire blowdown pressure range studied in this work. This point will be addressed in greater detail in the following sections.

\section{PDRE Performance Results}

The performance trends just discussed can be seen in greater detail by studying the performance of the various PDRE geometries over a wide range of blowdown pressure ratios. The specific impulse of the baseline detonation tube (geometry A), and the detonation tube with several extension lengths (geometry B with $L_{e}=4,10,20$, and $40 \mathrm{~cm}$ ) is shown in Fig. 7. As is evident from the figure, the general performance trends of all five configurations are quite similar. There is a fairly significant improvement in performance $(30-38 \%)$ over the blowdown pressure ratio range of 1-10, while decidedly less so (4-8\%) from 10-100. There is very little improvement in performance $(\sim 1 \%)$ over the blowdown pressure ratio range of $100-1000$. In general, there is a definite performance improvement provided by the extension section, and this benefit increases with extension length. The one exception to this trend occurs at a blowdown pressure ratio of two. In this particular case, there is no performance benefit for extensions

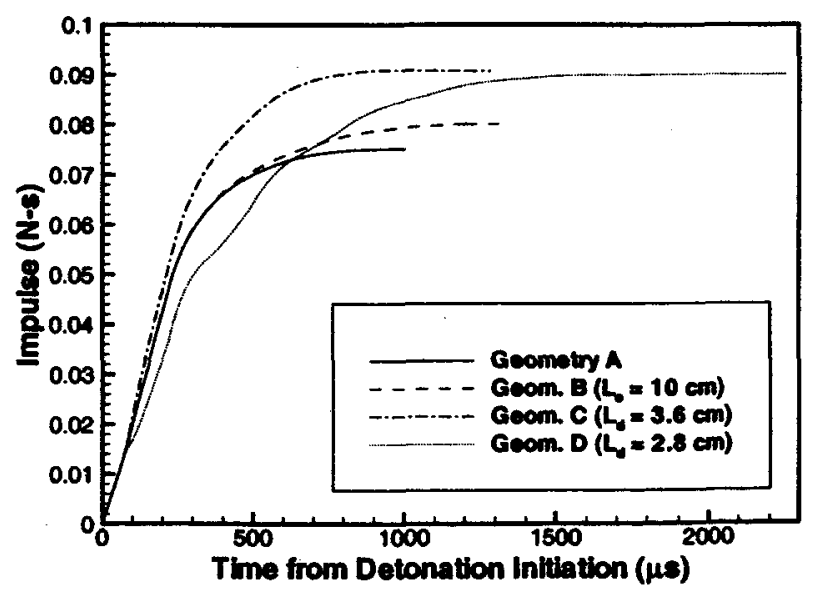

Fig 6 Impulse history for example PDRE geometries. Blowdown pressure ratio is $10\left(P_{\alpha}=0.1 \mathrm{~atm}\right)$. Propellant mixture: stoichiometric $\mathrm{H}_{2}-\mathrm{O}_{2}$. Propellant initial conditions: $\mathbf{P}_{c, i}=$ $1 \mathrm{~atm}, \mathrm{~T}_{c, i}=300 \mathrm{~K}$.

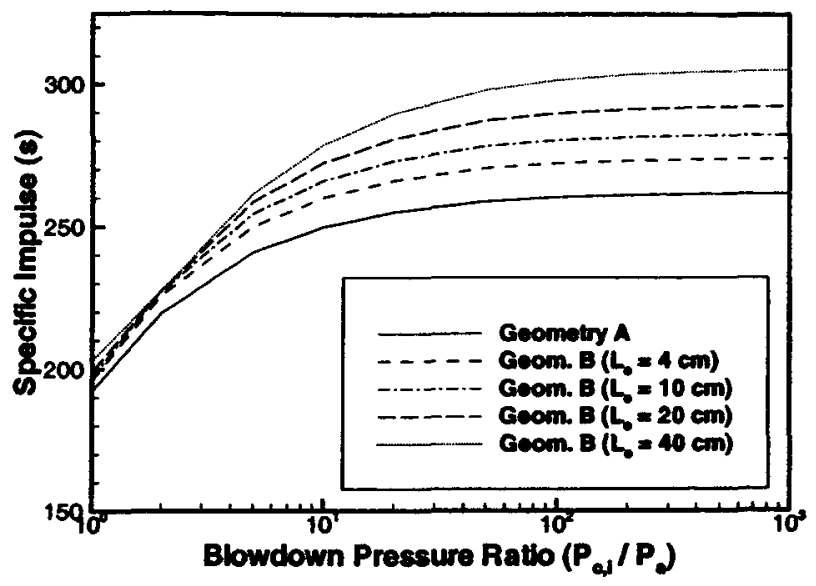

Fig. 7 Effect of blowdown pressure ratio on specific impulse for PDRE geometries A and B. Propellant mixture: stoichiometric $\mathrm{H}_{2}-\mathrm{O}_{2}$. Propellant initial conditions: $P_{c, i}=1 \mathrm{~atm}, T_{c, i}$ $=300 \mathrm{~K}$.

longer than $10 \mathrm{~cm}$. This is due to the fact that the primary expansion wave, generated at the interface between the detonation tube and the extension section, is alone sufficient to reduce the pressure at the end-wall to the ambient value. In this particular case, this event occurs before the secondary expansion wave (generated when the transmitted shock reaches the exit of the extension) can reach the endwall. Thus, there is no effect from the longer extensions at this pressure ratio. At higher blowdown pressure ratios (51000 ) this phenomena does not occur; the pressure decay (to ambient) provided by the primary expansion at the endwall is long enough that the secondary expansion wave can reach the end-wall in time to affect the pressure relaxation rate.

Both the blowdown pressure ratio, and the nozzle area ratio, play critical roles in the performance of the C-D nozzle PDRE geometries. Performance results as a function of C-D nozzle area ratio for geometries C and D (upper and lower panels, respectively) are shown in Fig. 8. In each case, the area ratio was adjusted by varying the length of the diverging section of the nozzle $\left(L_{d}\right)$. Results for blowdown pressure ratios of 1-1000 are plotted. As is the case for conventional rocket-type systems, for a given pressure ratio, the performance gain provided by a C-D nozzle will generally increase with area ratio up to a certain optimum point. After this point performance will decrease with increasing nozzle area ratio. The dashed curve in each graph shows the fixed nozzle area ratio which yields optimum specific impulse for each blowdown pressure ratio. It is important to recognize that this optimum point is specific to the C-D nozzle geometry and blowdown time assumption used.

In general, there is little performance difference between the two C-D nozzle geometries. The performance of geometry D, at a given area and pressure ratio, tends to be slightly lower than geometry C, primarily due to losses induced by the reflected shock waves present in the blowdown history of D. Note that a C-D nozzle is generally 

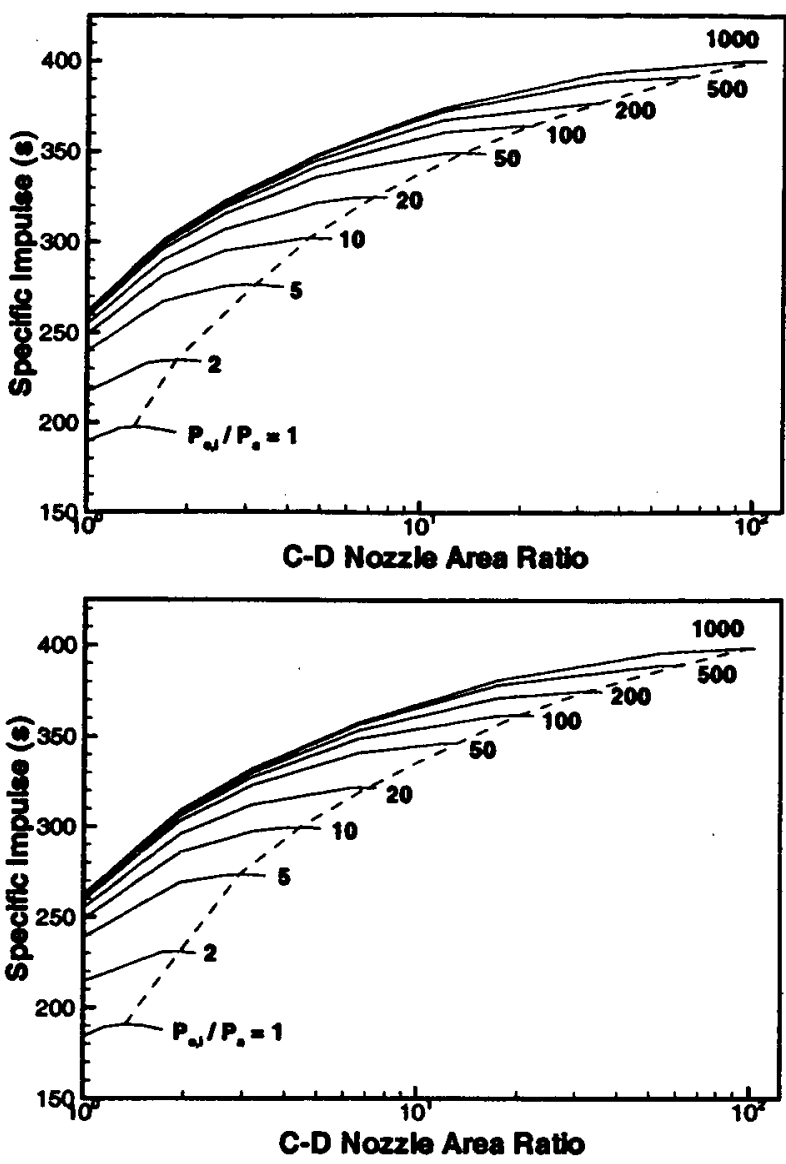

Fig. 8 Effect of C-D nozzle area ratio on specific impulse for PDRE geometry C (upper panel) and D (lower panel). The dashed line in each graph represents the fixed nozele area ratio giving optimum performance at each pressure ratia. Propellant mixture: stoichiometric $\mathrm{H}_{2}-\mathrm{O}_{2}$. Propellant initial conditions: $P_{c, i}=1 \mathrm{~atm}, T_{c, i}=300 \mathrm{~K}$.

more effective at improving performance at higher pressure ratios than the extensions in geometry $B$. The specific impulse using an optimized C-D nozzle improves by 53$57 \%$ over a blowdown pressure ratio range of $1-10$, and 20-21\% over 10-100. Even over a blowdown pressure ratio range of 100-1000, the performance increases $10 \%$. This is largely due to the ability of a C-D nozzle to convert the thermal energy of the combustion products into directed kinetic energy. This effect can also be seen by plotting specific impulse for all four geometries as a function of the ratio of total assembled tube volume to detonation tube volume, $V_{0} / V_{t}$ (Fig. 9). The blowdown pressure ratio is 1000 in the upper panel of the figure, and 10 in the lower panel. It is evident from the figure that a C-D nozzle is generally a more effective use of volume than a straight extension, particularly at relatively high blowdown pressure ratios. It should be noted, however, that this observation is based on the assumption that the volume ratio range under consideration is within the range where increased nozzle expansion provides improved performance.
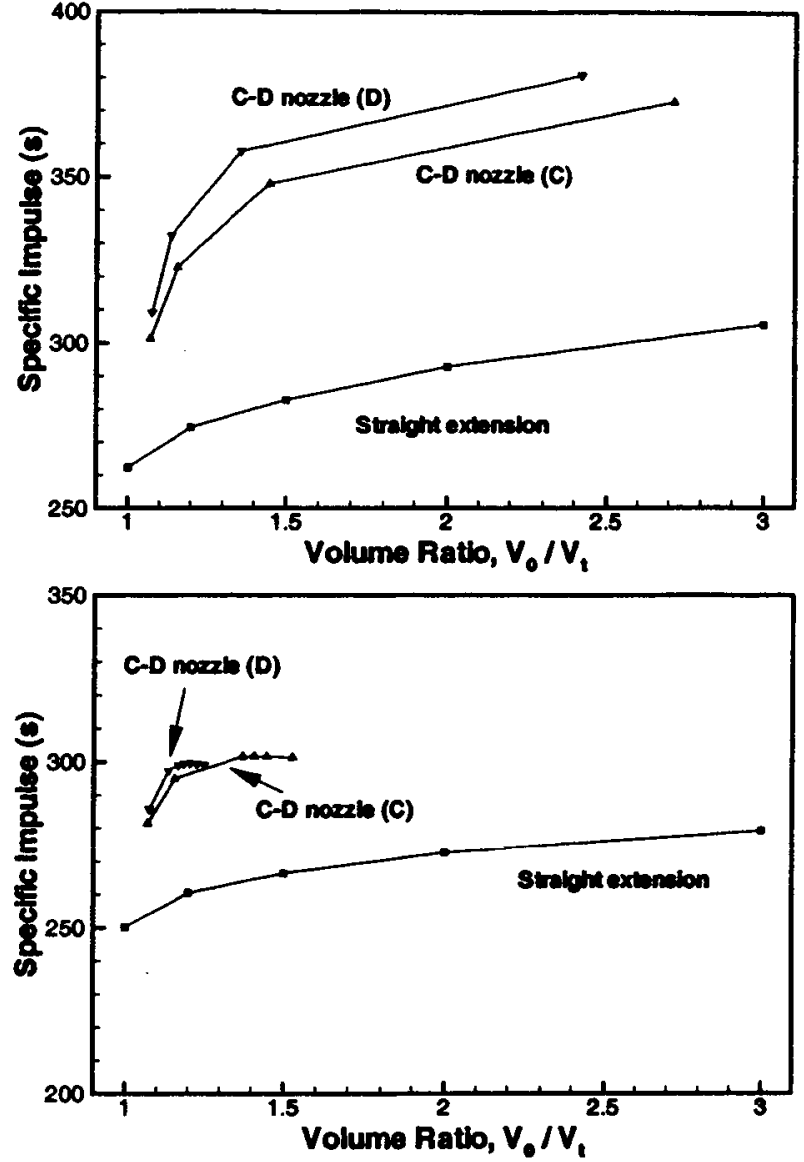

Fig 9 Specific impulse plotted as a function of the ratio of total assembled tube volume to detonation tube volume, $V_{o} / V_{t}$. Blowdown pressure ratio = 1000 (upper panel) and 10 (lower panel). Propellant mixture: stoichiometric $\mathrm{H}_{2}-\mathrm{O}_{2}$. Propellant initial conditions: $P_{c, i}=1 \mathrm{~atm}, T_{c, i}=300 \mathrm{~K}$.

\section{PDRE Blowdown Time Results}

As the cycle time of an operational PDRE system is of great practical interest, it is useful to examine the effect of the blowdown pressure ratio, straight extension length, and C-D nozzle area ratio on the blowdown time of the PDRE geometries considered here. As has been discussed previously, the stopping point for all simulations of this study is the time at which the pressure at the end-wall decays to the ambient value. For the purpose of this discussion, we will define the blowdown time, $t_{b d}$, as equal to this time value. The strong dependence of $t_{b d}$ on blowdown pressure ratio and extension length can be seen in Fig. 10. The time in that figure has been normalized by $U_{C-J} / L_{t}=70.495 \mu \mathrm{s}$, which is the characteristic time for a C-J detonation to traverse the detonation tube. Note that the highest blowdown pressure ratios, there is an almost directly proportional dependence of blowdown time on extension length. The obvious kink in the $L_{e}=20 \mathrm{~cm}$ and $L_{e}=40 \mathrm{~cm}$ curves at a blowdown pressure ratio of two was explained in the previous section.

The blowdown time for the two converging-diverging nozzle PDRE geometries is shown in Fig. 11. The values have again been normalized by $U_{C-J} / L_{t}$. As would 
be expected, $t_{b d}$ is consistently longer for geometry $\mathrm{D}$ than $\mathrm{C}$, over the entire range of blowdown pressure ratios. Note that these results assume the use of the optimum fixed CD nozzle for each pressure ratio, though since the nozzle throat is largely choked, $t_{b d}$ is nearly independent of $L_{d}$ at a given pressure ratio. One of the notable results of these simulations is that the instantaneous thrust of even an optimum fixed C-D nozzle is slightly negative for much of the latter portions of the blowdown time. This result is due to the fact that the C-D nozzle overexpands the residual combustion products when the pressure in the detonation tube has decayed sufficiently. Thus, it is useful to also plot the time at which the thrust first drops to zero in the blowdown history $\left(t_{z}\right)$. It is evident from the figure that this point occurs considerably earlier than $t_{b d}$, particularly at the highest pressure ratios. As the instantaneous thrust, even while negative, is so small at this point, there is little difference in integrated impulse between these two time points (typically at most a loss of $\sim 1 \mathrm{~s}$ of specific impulse over the interval). However, this observation suggests that a considerable savings in overall cycle time could be realized with an actual slight gain in performance. Note that as the end-wall is the only thrust surface for geometries A and $\mathrm{B}, t_{z t}=t_{b d}$ for those cases.

\section{Equivalent SSRE Model and Performance Comparison}

It is instructive to compare the specific impulse of a PDRE fitted with a C-D nozzle with a SSRE under equivalent modeling assumptions. While frozen and equilibrium rocket performance calculations can be readily obtained from the CEA code, these results are not directly comparable to the finite-rate chemistry model used in the current PDRE code. The primary concern is the tendency for chemistry to slow down in real nozzle systems as the temperature and pressure are reduced in the expansion process. Thus, it is best to compare the finite-rate PDRE systems with a finite-rate SSRE model. This SSRE CFD model is heavily derived from the PDRE code. The $20 \mathrm{~cm}$ det-

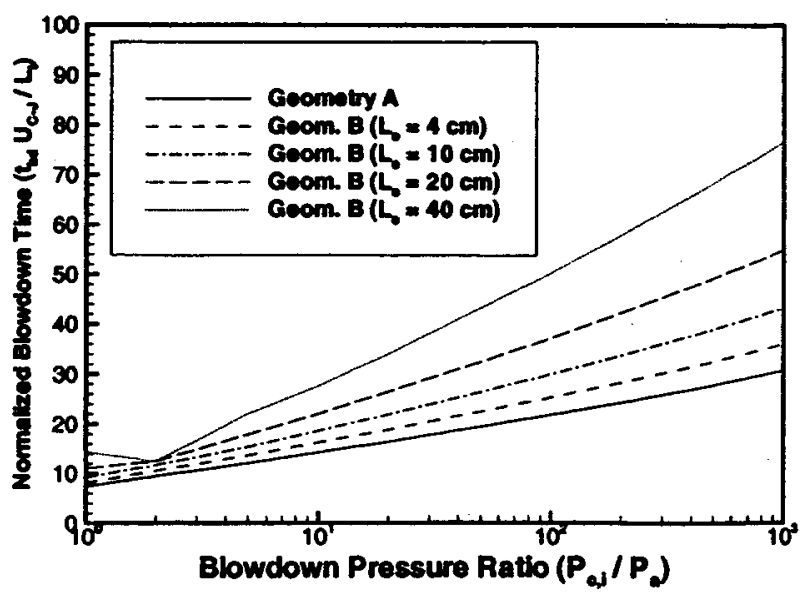

Fig. 10 Normalized blowdown time $\left(t_{b d} U_{C-J} / L_{t}\right)$ plotted as a function of blowdown pressure ratio for PDRE geometries $A$ and B. Propellant mixture: stoichiometric $\mathrm{H}_{2}-\mathrm{O}_{2}$. Propellant initial conditions: $P_{c, i}=1 \mathrm{~atm}, T_{c, i}=300 \mathrm{~K}$.

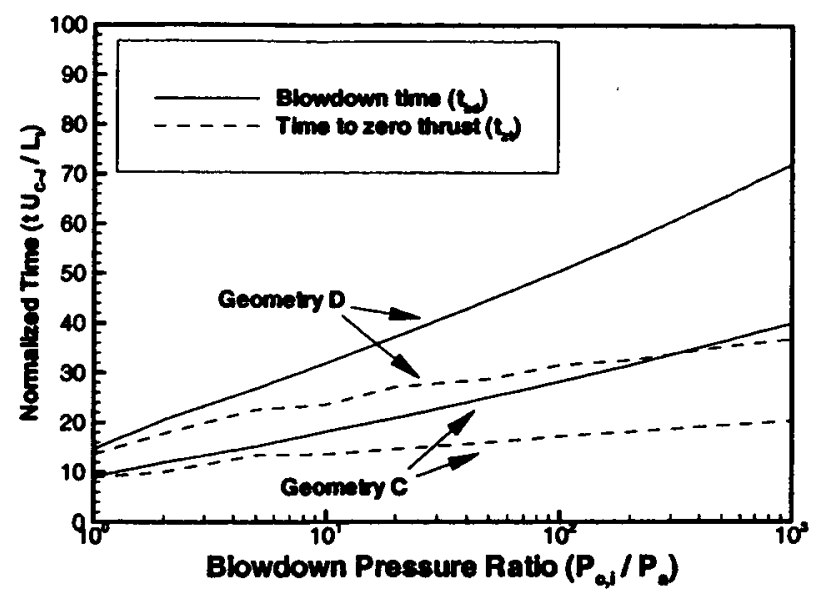

Fig. 11 Normalized time $\left(t U_{C-J} / L_{t}\right)$ plotted as a function of blowdown pressure ratio for PDRE geometries $C$ and D. Propellant mixture: stoichiometric $\mathrm{H}_{2}-\mathrm{O}_{2}$. Propellant initial conditions: $P_{c, i}=1 \mathrm{~atm}, \mathrm{~T}_{c, i}=300 \mathrm{~K}$.

onation tube is replaced with a thrust chamber $0.1 \mathrm{~cm}$ in length, and $4.0 \mathrm{~cm}$ in diameter (Fig. 12). This thrust chamber then converges at a constant $15^{\circ}$ angle, over a length of $4.13 \mathrm{~cm}$, to a throat $1.787 \mathrm{~cm}$ in diameter. After the throat, the nozzle diverges at $15^{\circ}$ to a length specified by the user. Thus, the throat diameter and nozzle expansion rate of the SSRE model closely matches that of PDRE geometry $C$. The equilibrium temperature, pressure and composition of stoichiometric $\mathrm{H}_{2}-\mathrm{O}_{2}$, burned at constant pressure and enthalpy from initial conditions of $300 \mathrm{~K}$ and $1 \mathrm{~atm}$, are fed as a constant enthalpy reservoir inflow boundary condition into the domain. Thus, this analysis assumes that the propellant feed system of the SSRE model is the same as the PDRE.

Similar to the PDRE nozzle optimization study, the finite-rate SSRE CFD model is run at various expansion ratios until the optimum specific impulse is obtained for a given pressure ratio. However, as the solution converges to steady-state in this model, flowfield information from the highest pressure ratio case can be used to guide area selection at the lower pressure ratios. In each case, the SSRE model is run until the solution converges. In general, the specific impulse and optimum expansion ratio using finite-rate chemistry are slightly larger than that for frozen chemistry. Additionally, if the chemistry is frozen in the SSRE CFD model, there is excellent agreement (to within $0.3 \%$ in specific impulse) with the frozen-chemistry predictions of CEA.

The mixture-based specific impulse for the baseline detonation tube (PDRE geometry A), PDRE geometry $\mathrm{C}$ fitted with an optimized fixed C-D nozzle (at each blowdown pressure ratio), and the equivalent optimized SSRE system is plotted for blowdown pressure ratios of $1-1000$ in Fig. 13. As would be expected from the results discussed previously, the relative gain from the C-D nozzle systems becomes more pronounced at higher pressure ratios. It is interesting to note that that both the baseline and C-D nozzle PDRE systems outperform a SSRE at pressure ratios 
below $\sim 7$. This is due to the fact that the relative gain from the extra pressurization provided by the detonation process is greatest in this regime. Thus, the PDRE may have considerable theoretical potential for rocket-type applications when the pressure of the ambient environment is high. Additionally, at higher blowdown pressure ratios, a PDRE with an optimized fixed C-D nozzle still has a greater specific impulse than an equivalent SSRE. These results indicate that a PDRE equipped with a fixed C-D nozzle, though obviously not as efficient at generating thrust as a (theoretical) dynamically adaptive nozzle, can still yield performance superior to an equivalent SSRE over a wide range of pressure ratios. Note, however, that this performance gain becomes relatively smaller at higher pressure ratios.

\section{Conclusions}

A quasi-1-D, finite-rate chemistry CFD model for studying PDRE gasdynamics and performance is described and implemented. The single-shot performance and blowdown time characteristics of four different PDRE geometries are studied, over a range of blowdown pressure ratios from 11000. The pressure at the end-wall is allowed to decay to the ambient value for all simulations in this work. The results show that both straight extensions and convergingdiverging nozzles can provide valuable improvements to the performance of a baseline detonation tube. However, optimized C-D nozzles are generally more effective in increasing performance than straight extensions, particularly at higher pressure ratios. Studies of the blowdown time of the PDRE systems show a strong dependence on pressure ratio for all geometries. Straight extension length will also generally increase blowdown time. Examination of the blowdown history of the optimized C-D nozzle geometries shows that the blowdown of these systems could be cut off well before the end-wall pressure reaches the ambient value, with an actual slight gain in performance. The performance of a PDRE fitted with an optimized fixed C-D nozzle is also compared with a SSRE using similar modeling assumptions. The results show that while a PDRE enjoys the largest relative performance gain over a SSRE at low pressure ratios, there is still a noticeable relative advantage for the PDRE at a blowdown pressure ratios of 1000.

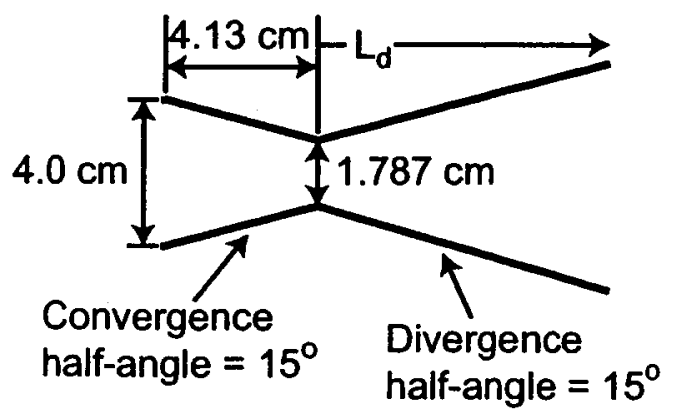

Fig. 12 Schematic of SSRE geometry studied.

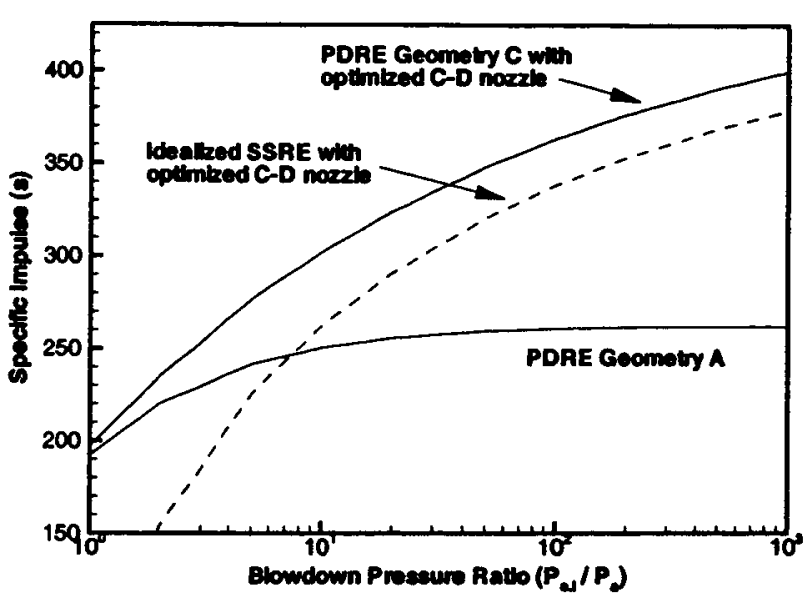

Fig 13 Performance comparison of various pulse detonation and steady-state rocket devices. All results obtained using finite-rate quasi-1-D CFD calculations. The final blowdown pressure at the end-wall in the PDRE is equal to the ambient pressure $\left(P_{c, f}=P_{a}\right)$. Propellant mixture: stoichiometric $\mathrm{H}_{2}-\mathrm{O}_{2}$. Propellant initial conditions: $P_{c, i}=1 \mathrm{~atm}, T_{c, i}=$ $300 \mathrm{~K}$.

\section{References}

'Kailasanath, K., "Recent Developments in the Research on Pulse Detonation Engines," AIAA Journal, Vol. 41, No. 2, 2003, pp. 145-159.

${ }^{2}$ Bratkovich, T. E., Aamio, M. J., Williams, J. T., and Bussing, T. R. A., "An Introduction to Pulse Detonation Rocket Engines," AlAA Paper 97-2742, July 1997.

${ }^{3}$ Sutton, G. P. and Biblarz, O., Rocket Propulsion Elements, John Wjley \& Sons, seventh ed., 2001, pp. 27-101.

${ }^{4}$ Kailasanath, K., "A Review of Reseanch on Pulse Detonation Engine Nozzles," AIAA Paper 2001-3932, July 2001.

${ }^{5}$ Cooper, M. and Shephend, J. E., "The Effect of Nozzles and Extensions on Detonation Tube Performance," AIAA Paper 2002-3628, July 2002.

${ }^{6} \mathrm{Li}, \mathrm{C}$. and Kailasanath, K., "Partial Fuel Filling in Pulse Detonation Engines," Journal of Propulsion and Power, Vol. 19, No. 5, 2003, pp. 908 916.

7 Cambier, J.-L., "Preliminary Modeling of Pulse Detonation Rocket Engines," AIAA Paper 99-2659, June 1999.

${ }^{8}$ Mohanraj, R. and Merkle, C. L., "A Numerical Study of Pulse Detonation Engine Performance," AIAA Paper 2000-0315, Jan. 2000.

${ }^{9}$ Mohanraj, R., Merkle, C. L., and Ebrahimi, H. B., "Modeling of Pulse Detonation Engine Operation," AIAA Paper 2001-0475, Jan. 2001.

${ }^{10}$ Talley, D. G. and Coy, E. B., "Constant Volume Limit of Pulsed Propulsion for a Constant $\gamma$ Ideal Gas," Journal of Propulsion and Power, Vol. 18, No. 2, 2002, pp. 400-406.

${ }^{11}$ Morris, C. I., "Simplified Analysis of Pulse Detonation Rocket Engine Blowdown Gasdynamics and Performance," AIAA Paper 2002-3715, July 2002

${ }^{12}$ McBride, B. J., Gordon, S., and Reno, M. A., "Coefficients for Calculating Thermodynamic and Transport properties of Individual Species," Tech. Rep. TM 4513, NASA, 1993.

${ }^{13}$ Petersen, E. L. and Hanson, R. K., "Reduced Kinetic Mechanisms for Ram Accelerator Combustion," Joumal of Propulsion and Power, Vol. 15, No. 4, 1999, pp. 591-600.

${ }^{14}$ Kee, R. J., Rupley, F. M., and Miller, J. A., “Chemkin-II: A FORTRAN Chemical Kinetics Package for the Analysis of Gas-Phase Chemical Kinetics," Tech. Rep. SAND89-8009, Sandia National Laboratories, 1989.

${ }^{15}$ Troe, J., "Predictive Possibilites of Unimolecular Rate Theory," Journal of Physical Chemistry, Vol. 83, No. 1, 1979, pp. 114-126.

${ }^{16}$ Strang, G., "On the Construction and Comparison of Difference Schemes," SIAM Journal of Numerical Analysis, Vol. 5, 1968, pp. 506517. 
${ }^{17}$ Oran, E. S. and Boris, J. P., Numerical Simulation of Reactive Flow, Cambridge University Press, 2nd ed., 2001, pp. 408-411.

${ }^{18}$ Yee, H. C., "A Class of High-Resolution Explicit and Implicit Shock-Capturing Methods," Tech. Rep. TM 101088, NASA, 1989.

${ }^{19}$ Grossman, B. and Cinnella, P., "Flux-Split Algorithms for Flows with Non-equilibrium Chemistry and Vibrational Relaxation," Journal of Computational Physics, Vol. 88, 1990, pp. 131-168.

${ }^{20}$ Larrouturon, B., "How to Preserve the Mass Fractions Positivity when Computing Compressible Multi-component Flows," Journal of Computational Physics, Vol. 95, 1991, pp. 59-84.

${ }^{21}$ Poinsot, T. J. and Lele, S. K., "Boundary Conditions for Direct Simulations of Compressible Viscous Flows," Jowrnal of Computational Physics, Vol. 101, 1992, pp. 104-129.

${ }^{22}$ Gordon, S. and McBride, B. J., "Computer Program for Calcula. tion of Complex Chemical Equilibrium Compositions and Applications," NASA Reference Publication 1311, 1994. 